\title{
Ventriculosubgaleal shunt procedure and its long-term outcomes in premature infants with post-hemorrhagic hydrocephalus
}

\author{
Vaner Köksal • Suat Öktem
}

Received: 28 December 2009 / Accepted: 19 February 2010/Published online: 19 March 2010

(C) The Author(s) 2010. This article is published with open access at Springerlink.com

\begin{abstract}
Objective It is well known that $10-15 \%$ of hydrocephalus cases at childhood and $40-50 \%$ in premature infants, occur following Germinal matrix hemorrhage $(\mathrm{GMH})$. Such hemorrhages are reported to arise due to the rupture of germinal matrix $(\mathrm{GM})$ vessels as a result of cerebral blod flow changes among infants with $<1500 \mathrm{~g}$ birth weight and $<32$ weeks old. Intraventricular hemorrhage (IVH) associated with GMH leads to a disruption in the cerebrospinal fluid (CSF) and ventricular dilatation. Ventriculosubgaleal shunt (VSGS) is preferred in those hydrocephalus cases because it is a simple and rapid method, precludes the need for repetitive aspiration for evacuation of CSF, establishes a permanent decompression without causing electrolyte and nutritional losses, and aims to protect the cerebral development of newborns with GMH.

Material and method The present study comprises 25 premature cases, subjected to VSGS and diagnosed with post-hemorrhagic hydrocephalus ( $\mathrm{PHH}$ ) arising from IVH associated with GM, and low birth weight (LBW) in the Neurosurgery Department of the Medical Faculty of Erciyes University between July 2002 and September 2006. VSGS surgery was performed on those cases, and their clinical and radiological prognoses were monitored with regard to several parameters.

Results Mortality and morbidity results were found to be lower than those in $\mathrm{PPH}$ treatment methods. While
\end{abstract}

\footnotetext{
V. Köksal $(\bowtie)$

Rize 82. year Government Hospital, Neurosurgery Clinics, Kayseri, Turkey

e-mail: vanerkoksal@hotmail.com

S. Öktem

Department of Neurosurgery, Erciyes University Medical School, Kayseri, Turkey

e-mail: oktemis@hotmail.com
}

prognosis of grade 4 GMHs was poor, grades 2 and 3 GMHs displayed a much better prognosis after VSGS along with complete recovery in some hydrocephalus cases.

Keywords Germinal matrix hemorrhage $\cdot$ Hydrocephalus . Ventriculosubgaleal shunt

\section{Introduction and objective}

Currently, there is still no consensus over the optimal treatment of hydrocephalus cases occurring as a result of germinal matrix hemorrhage among premature infants with low birth weight. Therefore, this patient group, comprises the cases that are most difficult to treat in Intensive Care Units. In hydrocephalus treatment required at that period, applying VSGS prior to the placement of a permanent shunt, is reported to be beneficial. In light of this recommendation, we aim to investigate VSGS treatment in cases with post-hemorrhagic hydrocephalus and its contribution to the hydrocephalus prognoses.

\section{Material and method}

The present study comprises 25 premature cases, subjected to VSGS and diagnosed with post-hemorrhagic hydrocephalus $(\mathrm{PHH})$ arising from IVH associated with GM and LBW in the Neurosurgery Department of the Medical Faculty of Erciyes University between July 2002 and September 2006. Those cases were subjected to surgery 28 times, among which, the procedure was repeated due to VSGS dysfunction in one case and because of meningitis in two cases.

GMH and hydrocephalus diagnoses of the cases were established in light of the routine ultrasonography (US) 
performed in the newborn unit. GMHs were studied in four grades based on the classification (Fig. 1) of Papile et al. [1].

Grade 1. Hemorrhage is limited with the germinal matrix Grade 2. There is an IVH but no ventricular dilatation

Grade 3. There is IVH associated with ventricular dilatation

Grade 4. There is a diffuse parenchymal hemorrhage

As a result of the monitoring of the head circumferences (HC) of the cases; in cases who exhibited an enlargement of more than $2 \mathrm{~cm}$ and tense fontanelles along with apnea and bradycardia, VSGS operation was decided upon determination of ventricular dilatation by US. All the cases diagnosed by US were monitored by brain computed tomography.

1. Gender

2. Birth age (weeks)
3. Birth weight (BW)

4. Head circumference measurements were measured at birth, before and after VSGS, and before and after VPS.

5. GMH were classifed based on grades.

6. Exposure to mechanic ventilation and asphyxia were investigated since it elevates cerebral ischemic damage.

7. VSGS and VPS timings are determined according to the clinical and radiological findings.

8. Complications during VSGS including CSF leak from incision site, migration of catheter to the subgaleal pouch, slippage of the catheter into the ventricle, development of meningitis, and intraparenchymal and subdural hemorrhage, were investigated.

9. Clinical status after the clinical interventions was classifed in three different groups depending on the

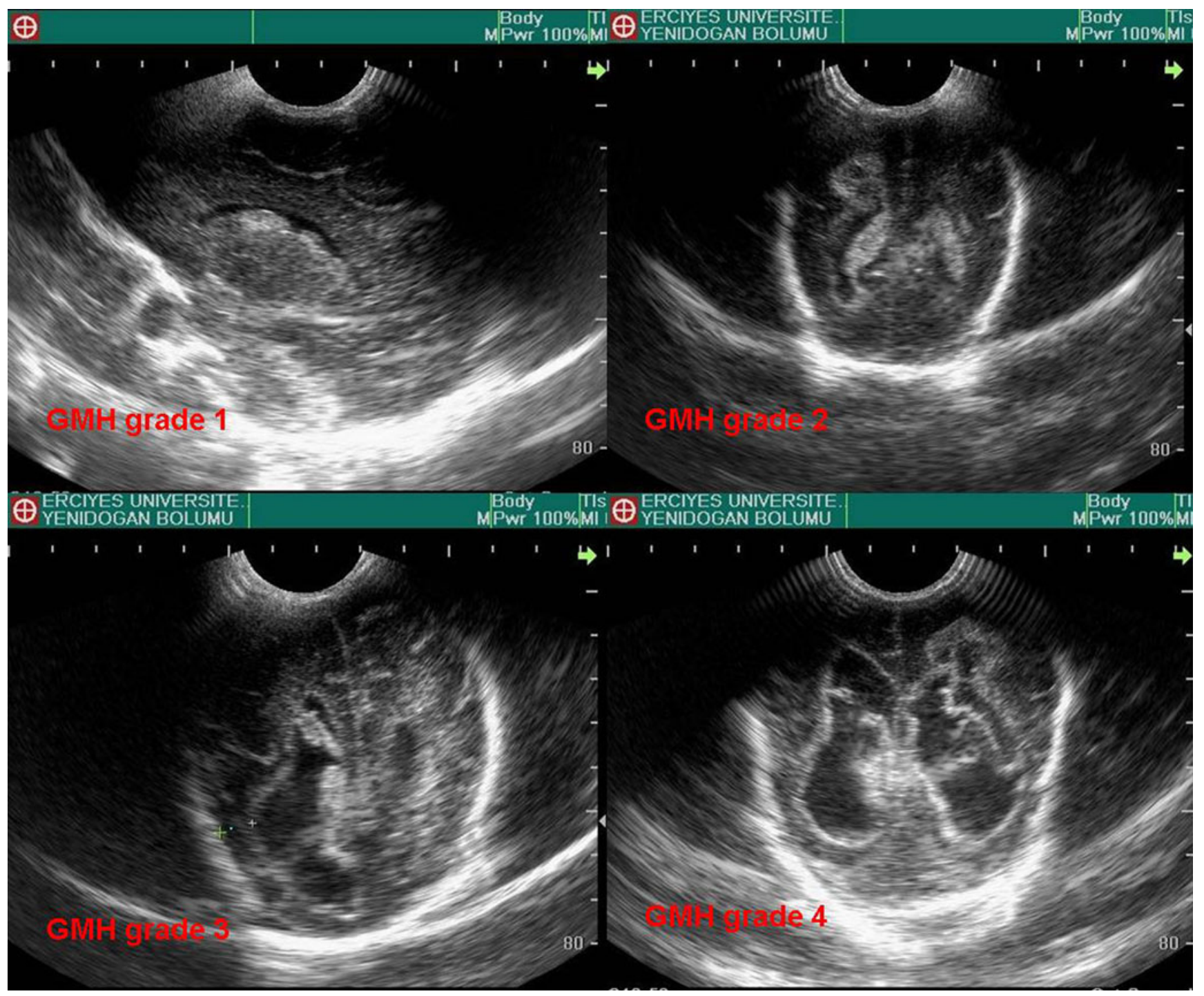

Fig. 1 Ultrasound images with the classification of Papile et al. 
neurological conditions of the survived cases: a good overall condition (ones with a good overall health), mild neurological sequelae (mildmotor paresias), and severe neurological deficits (e.g. inability to hold one's head upright, sit, presence of a motor deficit as plegia, etc.). The reasons behind mortality were investigated in lost cases.

10. The cases were monitored radiologically by computed tomography (CT); Changes in the cerebral cortices were observed on CT images taken after VSGS and VPS (Fig. 2).

The method of ventriculosubgaleal shunt

Subgaleal shunt procedure was performed after $2-3 \mathrm{~h}$ of fasting, under $1-2$ cc local anesthesia (2\% prilocaine hydrochloride) and mild sedation (1.5\% sevoflurane) without intubation and by nasal oxygen support in operating room settings or intensive care unit (two cases).

Approximately $1.5 \mathrm{~cm}$ skin incision was used from the right corner of the anterior fontanelle (Fig. 3). Periosteum was reached. Following the determination of periosteum, subgaleal dissection was started with instruments having a blunt tip. As a result of the dissection, an area of at least $10 \times 10 \mathrm{~cm}$, which was located towards the temporal and parietal bones, extending to the most distant ear lobe laterally and vertex posteriorly, was reached. Thus, a large pocket was established by separating galea from the periosteum (Fig. 3(3)).

The margin of the incision was pulled towards the fontanelle as to expose the dura, and the vascular structures localized in the route of the ventricular catheter to be inserted, were cauterized by bipolar cauterization (Fig 3(4)).
A hole was opened by a small incision, and a ventricular catheter, approximately $3-\mathrm{cm}$ long, was inserted into the right lateral ventricle (Fig. 3(6)). The catheter was fixed to the dura, cut off 5-6 cm externally, and placed into the subgaleal pouch without using a metal instrument (Fig. 3(8)). No valve was employed during the procedure (Fig. 3(9)). The patients were not allowed to lay on the right temporal surface in order to encourage subgaleal collection. Depending on the condition of the scar, sutures were removed postoperatively at 8-10 days.

1. Tension in subgaleal fluid pouch (Fig. 4)

2. Poor subgaleal collection

3. An increase beyond physiological limits in $\mathrm{HC}$

4. No increase in $\mathrm{HC}$, but elevation in ventricle volume shown by imaging methods.

5. Clinical presence of intracranial pressure rise.

6. Persistence of hydrocephalus from prematurity to maturity.

For VPS procedure, burr hole and the same incision or a new posterior one, were used. In cases that received VPS posteriorly, the former anterior catheter was removed through a small incision on the scar. General prognosis and particularly the dependency for shunt were investigated according to the clinical monitoring of the cases that received VSGS. Windows SPSS program was used along with Chi-square and Spearman correlation tests.

\section{Results}

The present study comprises 25 premature cases, subjected to VSGS, and diagnosed with $\mathrm{PHH}$ arising from IVH associated with GM and LBW in the Neurosurgery Department of the
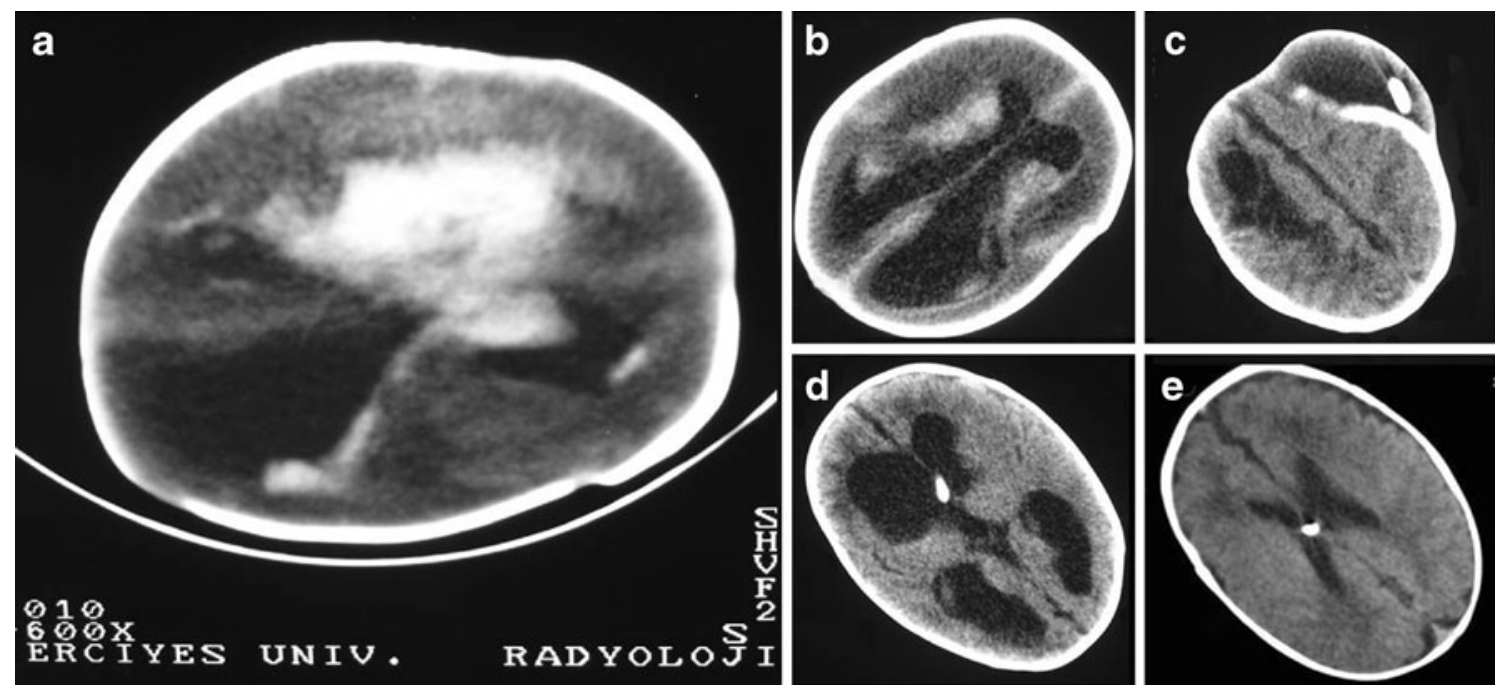

Fig. 2 Changes in the cerebral cortices were observed on CT images a. pre op b. 1 month after hemorrhage cases c. tomography subgaleal collection view $\mathbf{d}$. after 1 month post op e. after 2 year post op 

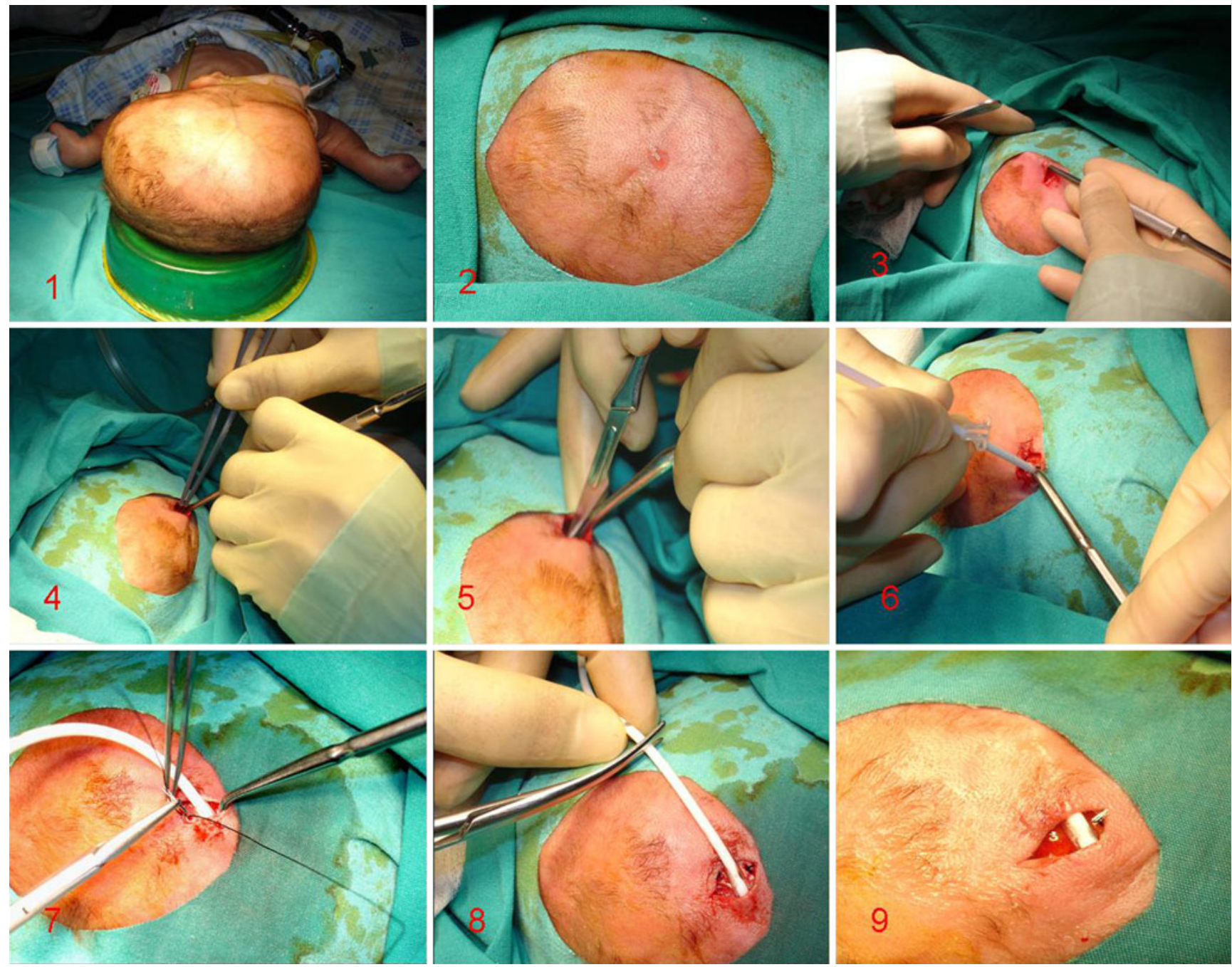

Fig. 3 Stages of the subgaleal shunt technique

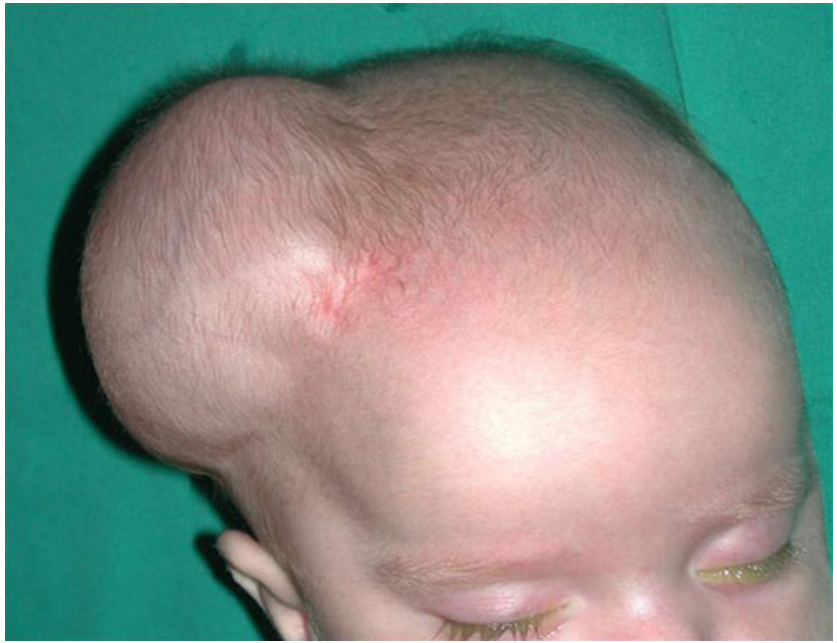

Fig. 4 Tension in subgaleal fluid pouch
Medical Faculty of Erciyes University between July 2002 and September 2006. Seventeen (68\%) of our cases were female and $8(32 \%)$ were male. In terms of age groups, two were 25 26-week-old, eight 27-28-week-old, five 29-30-week-old, five 31-32-week-old, and again five 33-34-year-old, whereas the mean birth age was 29.2 weeks. Birth weights varied between 740 and 1,930 g (mean 1,342 $\pm 338 \mathrm{~g}$ ). Based on monitoring values, VAs prior to VPS, varied between 1,900 and 4,300 g $(2,434 \pm 615 \mathrm{~g})$ (Fig. 5).

The head circumferences of the cases varied from 29.5 to $39.5 \mathrm{~cm}$ at birth (mean $32.89 \pm 2.50 \mathrm{~cm}$ ). HCs of the cases before VSGS procedure, varied between 33.5 and $40.0 \mathrm{~cm}$ (mean: $37.42 \pm 1.70 \mathrm{~cm}$ ), whereas, during VPS procedure, HCs were found to be between 33 and $45 \mathrm{~cm}$ (mean 38.69土 $3.27 \mathrm{~cm})$.

The cases that were being monitored in the premature unit and showing abnormal increases in terms of $\mathrm{HC}$, were subjected to ultrasonography (US). The cases with a detected GMH, were graded according to the classification 


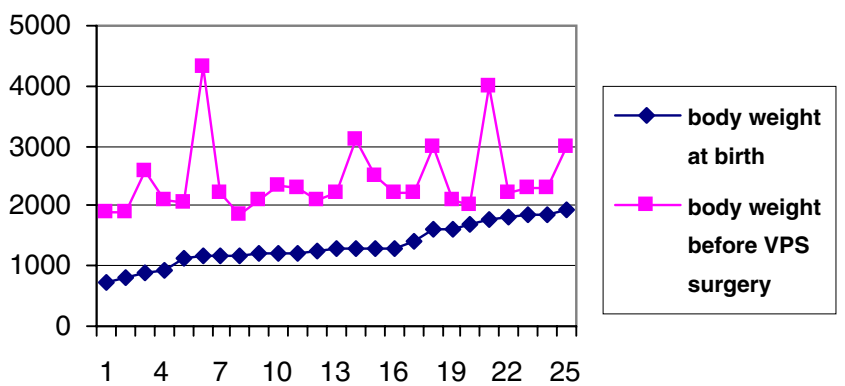

Fig. 5 Body weights of cases at birth and before VPS surgery

of Papile et al. as follows: 1 with no grade, 8 grade 2, 13 grade 3, and 4 grade 4. Eight of the cases had experienced asyphyxia at birth. Among those eight cases, four demonstrated grade $4 \mathrm{GMH}$, whereas the other four exhibited grade $3 \mathrm{GMH}$.

After stabilization of the vital signs of our cases within a period of 16-80 days (mean 33.08 \pm 15.4 days), VSGSs were placed. After that, VPS was performed on 15 cases at the end of a period varying between 27 and 92 days (mean: $44.53 \pm 17.31$ days). No intervention was applied in three cases that did not receive VPS due to resolution of the clinical profile of hydrocephalus. The three cases without shunt were observed to demonstrate no hydrocephalus recurrence. The remaining seven cases without shunt, died. Three VPS cases out of 15 did not show any VPS dysfunction, whereas six of the remaining 12 cases displayed shunt meningitis, and the other six received renewal of ventricular catheters due to shunt obstruction. Among cases with meningitis development, we removed VPS and waited until the stabilization of the CSF profile with extraventricular drainage (EVD). Three of the cases with shunt meningitis demonstrated recurrence.

Eight (32\%) of the VSGS cases were found to have no adequate CSF collection in the sulgaleal pouch during monitorization. In three of those, adhesions within the subgaleal space were cleaned, and a new subgaleal pouch was created. Thus, since two of those three cases were receiving meningitis treatment, their ventricular catheters were renewed, as well. Other five cases with no collection were subjected to VPS by assuming that they have reached the adequate body weight.

Postoperatively, none of the cases were let to lie on the dissection site in order to prevent re-adhesion of subgaleal pocket and help maintenance of adequate CSF drainage. However, because of lying on the same hemisphere, an increase in the anteroposterior (occipitofrontal) diameter of the cranium was observed similar to that seen in cases with sagittal synostosis. Therefore, craniums were immobilized by placing them onto circular pads created manually from dressings and cotton. Thus, the spherical form of the cranium was maintained.
Among cases with VSGS, seven (28\%) had CSF leak from the incision site, whereas one ( $4 \%$ ) had migration of catheter from the ventricle, one had slippage of catheter into the ventricle, and two (8\%) had meningitis. Meningitis cases were also the cases demonstrating CSF leakage. One case exhibited subdural hemorrhage at postoperative 15 days. In the case with a catheter migrated into the ventricle, only the catheter was removed due to arrested hydrocephalus.

Following VSGS, the patients were monitored as inpatients in the respective departments for 1-6 months and then as outpatients during 4-24 months. Seven of the cases were lost during the newborn unit care. Causes of mortality are outlined in Fig. 6. No mortality was observed at surgery.

As a result of the 2-year monitorization of the cases and in light of the general neurological examination results of the survivors, six cases were found to have mild neurologic sequelae, whereas the remaining six were found to exhibit severe neurologic sequelae. During follow-up, six cases with resolved hydrocephalus had normal cerebral cortex thickness along with presence of grade $2 \mathrm{GMH}$. In patients with mild neurologic deficits, ventriculomegaly appearance was still present. The cases with heavy neurologic deficits had irregular ventricle contours and loculations filled with cystic CSF (Fig. 7).

\section{Statistical results}

The results were analyzed with respect to the correlation between GMH grades and survival $\left(\chi^{2}=4.33, p>0.05, n=25\right)$. Our study had no grade 1 case. Of the grade 2 cases, $100 \%$ survived, whereas $38.5 \%$ of grade 3 cases were lost and $61.5 \%$ were survived. $50 \%$ of grade 4 cases were lost, while $50 \%$ survived first, but lost in the following days. According to the correlation analysis $(r=-0.415, p<0.05)$, the survival rate was found to be reduced as the grade elevated.

The correlation between hydrocephalus prognosis and GMH grades was analyzed, as well $\left(\chi^{2}=5.264, p>0.05\right)$. While $12.5 \%$ of grade 2 cases showed an increase in ventriculomegaly, $50 \%$ showed a normal cerebral cortex devleopment. As $38.5 \%$ of grade 3 cases exhibited an increase in ventriculomegaly, the increase in cerebral cortex thickness was $15.4 \%$. Of the grade 4 cases, $50 \%$ demon-
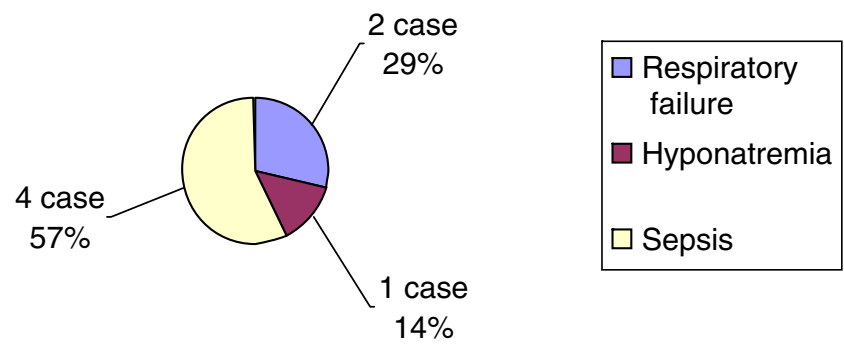

Fig. 6 The causes behind mortalities after VSGS procedure 
Fig. 7 Summary of cases that received VSGS procedure

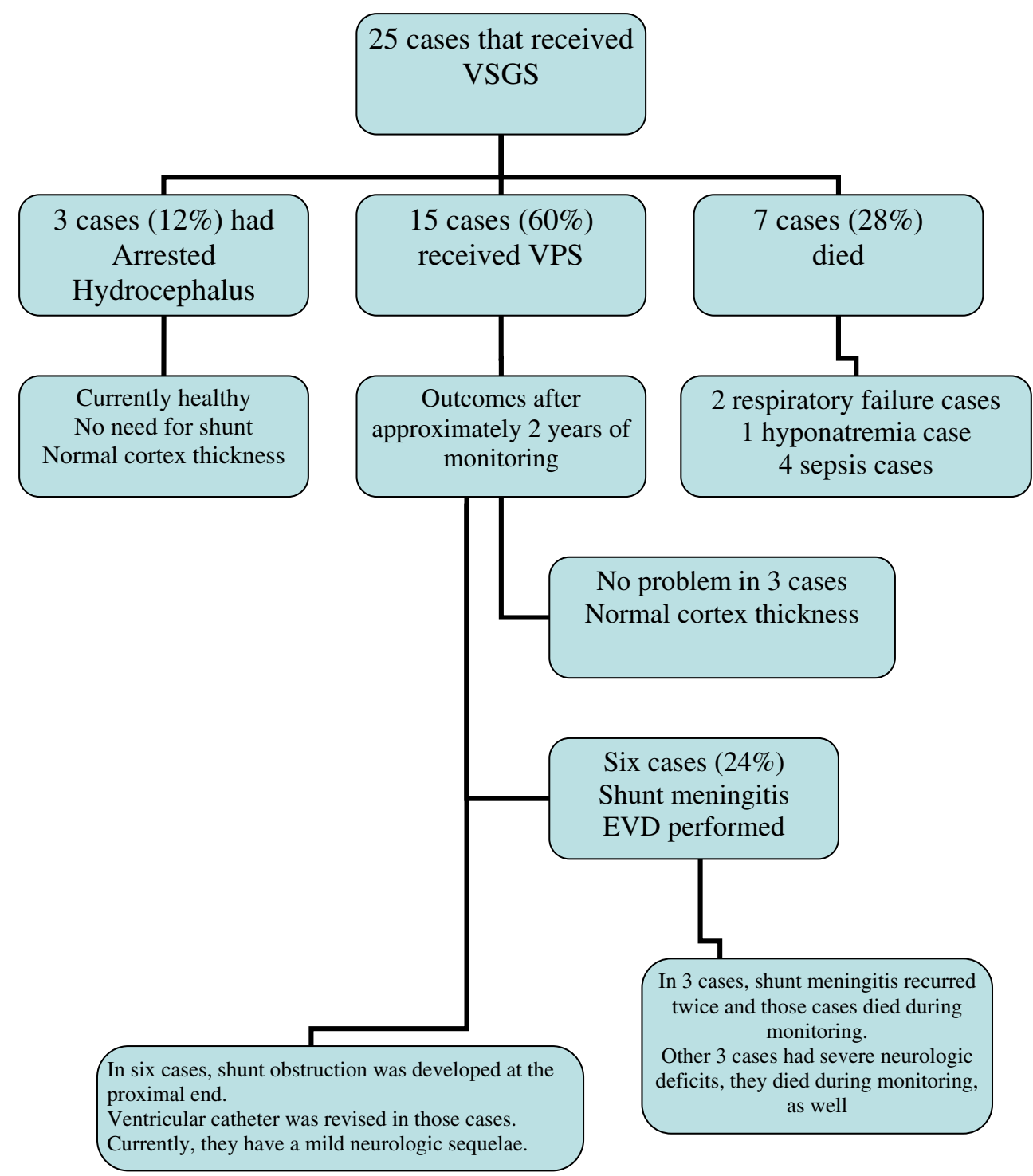

strated ventriculomegaly, whereas none of the cases had cerebral cortex development. In the correlation analysis ( $r=-0.426, p=0.034$ ), the prognosis was observed to worsen as the grade elevated.

While $25 \%$ of cases with a body weight (BW) of $500-1,000 \mathrm{~g}$ were observed to survive, $77 \%$ of cases that were $1,000-1,500 \mathrm{~g}$, and $87.5 \%$ of cases that were $1,500-2,000 \mathrm{~g}$ were found to survive, as well. No statistically significant correlation was determined between the BW and survival ( $r=$ $0.27, p<0.05)$. As the BW at birth rises, survival elevates.

\section{Discussion}

Currently, in hydrocephalus treatment, shunt systems that enable drainage of CSF to another body cavity in order to reduce the intracranial pressure caused by the collection of CSF fluid in the ventricle, are the most widely preferred methods. However, new treatment options are investigated because premature newborns demonstrate more complications associated with VPS systems. Premature newborns constitute the patient group that requires the most difficult care. VSGS procedure is known to be preferred in those cases due to restoration of permanent ventricular decompression without causing electrolyte and nutritional losses [2]. Treatment of hydrocephalus occurring after IVH has been tested with various methods by many clinical studies. However, although known for more than 100 years, VSGS procedure is observed to be employed at a much less rate compared with the others [3].

\section{Why VSGS (ventriculosubgaleal shunt)?}

Despite the consideration that adequate CSF drainage might not be achieved by VSGS even in premature cases with low 
birth weight, having a cerabral cortex thickness below $1 \mathrm{~cm}$ along with large ventricles on the ultrasound image, ventriculoperitoneal shunt (VPS) was not preferred over VSGS due to the following prerequisites needed for the VPS procedure: presence of a mature immune system and an adequate absorption capacity of the abdomen, effective elimination of blood products from the CSF flow in the ventricular system, and sufficient thickness of subcutaneous tissue. During the improvement of those parameters, the most important parameter that can be monitored is body weight. Therefore, it is commonly agreed that body weight should be waited to surpass $2,000 \mathrm{~g}$ before attempting the procedure $[2,4]$. During that period, a permanent solution with minimal damage potential is needed to protect the weakened cerebral parenchyma from the effects of elevated intracranial pressure and to prevent possible complications.

It is known that repeated ventricular taps and lumbar punctures in premature newborns, might increase the risk for meningitis. Similarly, external ventricular drainage systems expose the intraventricular environment to the exterior, and subsequently those cases with weak immune systems are subjected to infection risk. Moreover, they may lead to overdrainage problems. It is even reported that those three methods could cause metabolic disorders associated with the loss of CSF, protein, and electrolytes.

Furthermore, the subcutaneous ventricular reservoir system has been found to be an inappropriate method for premature infants without subcutaneous tissue. Because of repeated punctures from the reservoir aiming for aspiration, the skin covering the reservoir can be easily damaged. It is reported to be frequently complicated with infection and necrosis on the skin $[5,6]$.

Timing of VSGS practice on premature infants with hydrocephalus is a contentious issue in the literature with only two studies stating clear opinions. Willis et al. report this duration as 30 days (4.2 weeks), whereas Fulmer et al. express it as 28 days (4 weeks). In the present study, VSGS was performed after an average period of 35.04 days following birth. The average value was elevated due to poor vital signs of some patients and their care with ventilator support.

Performing VPS at an early period on premature PHH cases is reported to be associated with elevated shunt infection rates alongside high shunt obstruction rates [7]. Taylor et al. published a series of 36 cases in 2001 and advocated that VPS should be performed late on premature infants because of the need for clearance of ventricle from blood products which takes at least 5 weeks. Because VPS was applied after a mean period of 42 days on their cases and nine $(25 \%)$ of the 36 cases demonstrated a shunt obstruction, they had to apply 21 revisions [8]. They did not perform any procedure during that 42 days in order to protect the white matter around the ventricle from increas- ing intracranial pressure. However, grade 4 premature infants may show abrupt changes in their clinical conditions, and hydrocephalus can develop very rapidly [7, 9]. Under these circumstances, early VPS procedure is recommended $[2,3,7,8]$. Authors suggest that VPS should not be applied until the baby reaches the $2,000 \mathrm{~g}$ body weight, and CSF level decreases below $1,000 \mathrm{mg} / \mathrm{dl}[3,8]$.

Similarly, Levy et al. performed VPS in PHH treatment of premature cases and reported $83 \%$ shunt obstruction, whereas Lin JP et al., McCallum et al., and Scarff et al. noted shunt obstruction rates as 89,94 , and $50 \%$, respectively [11-15]. In the present study, $24 \%$ of our cases demonstrated an obstruction at the proximal end of the VPS after the procedure and those catheters were replaced. Obstruction complication of ventriculoperitoneal shunt in PHH treatment is observed to have a lower incidence because it is performed after VSGS.

In the literature, when VSGS fails in treatment of the hydrocephalus among premature cases with LBW and $\mathrm{PHH}$, VPS practice is reported on patients that are considered to reach the adequate maturity $[5,9,10,16]$. Tubbs et al. [17] reported the VPS timing or VSGS stay length as 37.4 days, whereas Fulmer et al. advocated this length to be at least 1 month in newborns with IVH because of the need for clearence of intraventricular debris [16, 17]. In the present study, VPS was performed after a mean period of 44 days. It may appear to be late according to the literature data, however, we waited for our cases to reach the 2,000 g body weight.

In the literature, complications seen during monitorization of cases with VSGS are CSF leakage from the incision site, meningitis, migration of the catheter from the ventricle or its slippage into the ventricle, and intraparenchymal hemorrhage [5, 9]. The rate of CSF leakage from the incision site has been reported to be $16.6 \%$ by Willis et al. [9], 4.7\% by Tubbs et al. [17], $5 \%$ by Fulmer et al. [5], and $32 \%$ by Sklar et al. [18]. In the present study, the rate of CSF leakage from the incision site was 29\% (seven cases), which was within the upper limit of the mean value of results reported in the literature. We believe, by paying more attention to the surgical closure technique, this complication may be reduced.

The rate of infection complication associated withVSGS is reported to be $66.7 \%$ by Willis et al. [9], $5.9 \%$ by Tubbs et al. [17], $0 \%$ by Fulmer et al. [5], and $10 \%$ by Sklar et al. [18]. Rahman et al. [19] reported no infection development after carrying out VSGS and VPS procedures on 15 premature infants with $\mathrm{PHH}$ and a birth weight below $1,500 \mathrm{~g}$. Moreover, in the absence of VSGS treatment, the infection rate after early VPS treatment in premature infants with PHH was found to be increasing from 20 to $50 \%$ by Vinchon et al. [20] and as $45.2 \%$ by Reinprecht et al. [2]. Infection rates of other methods used in $\mathrm{PHH}$ treatment in 
large series in the literature are reported to be 15.9-16.4 [21] in subcutaneous reservoir method and $10-27 \%$ in EVD method [22]. Richard et al. [23], placed Ommaya reservoir in 64 cases and reported complications (pulmonary problems, meningitis, sepsis and hemorrhage) in 30 of them, whereas noted the overall infection rate as $21.8 \%$. In the present study, infection rate was $8 \%$, which was consistent with the literature data on VSGS. Moreover, our result was found to be considerably lower than the infection rates of other treatment methods reported in the literature [20-24].

Other complications are rarely mentioned in the literature. Development rate of a new intraparenchymal hemorrhage has been noted to be $1.1 \%$ (two cases) by Tubbs et al. and 5\% (one case) by Fulmer et al. [5].

Moreover, mortality associated with shunt has been noted to be a result of that during which hemorrhage occurs because of the rupture of fragile corticomeningeal arteries and veins due to rapid intraoperative decompression in the weakly myelinized neonatal brain under pressure [5]. However, Fulmer et al. [5] and Tubbs et al. [17] noted the multifocal origin of intracerebral hemorrhage in their studies. While Fulmer et al. did not use valve in their VSGS procedures, Tubbs et al. [17] employed distal slit valve that enables one-way CSF flow to the subgaleal pouch, and suggested that intraparenchymal hemorrhage could be associated with that. Newborns subjected to VPS may present with that rare complication as a result of acute ventricular decompression [5, 17]. Moreover, Richard et al. provided fibrinolytic treatment to 17 cases in their study and reported four mortalities due to fatal diffuse hemorrhage [23]. In the present series, no intraparenchymal hemorrhage complication was observed after the VSGS procedure because no valve was inserted into the used catheters, and the wound was closed before allowing excessive CSF drainage. Moreover, one of our cases demonstrated a cerebral subdural hemorrhage that was observed 15 days after the VSGS (Fig. 8). Prior to the insertion of the ventricular catheter, because of inadequate cauterization of the dura it will pass through and the gradual venous hemorrhage in the newborn with a low intracranial pressure, formation of chronic subdural hematoma was considered. No such complication has been described in the literature.

Slippage of the catheter into the ventricle or its migration from the ventricle has been reported only by Fulmer et al. [5] in one (5\%) case. In the current study, similarly, one (4\%) case exhibited slippage of the catheter into the ventricle, whereas in another case the entire catheter migrated into the subgaleal space. Fulmer et al. [5] removed the catheter that slipped into the ventricle and performed a VPS procedure. In our case, the catheter was removed under endoscopic guidance, and by considering the hydrocephalus arrested, no other shunt intervention was found to

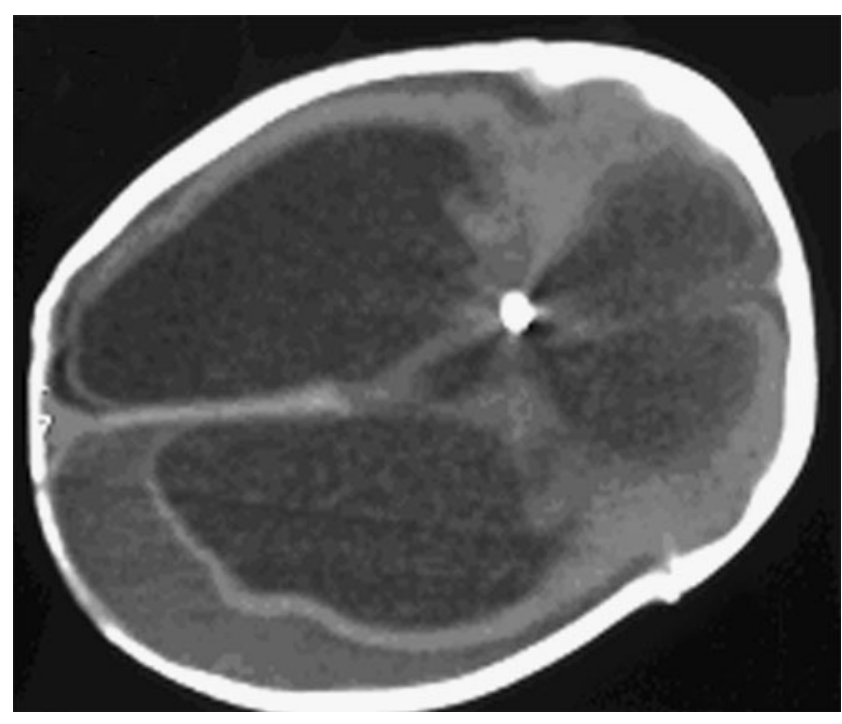

Fig. 8 Subdural hemorrhage, a complication

be necessary (Fig. 9). We carried out VPS in the other case where the catheter was migrated into the subgaleal space.

In the literature, the rate of mortality for cases with VSGS is reported to be $16 \%$ by Willis et al. [9], $9 \%$ by Tubbs et al. [17], $12 \%$ by Sklar et al. [18], and $20 \%$ by Fulmer et al. [5]. However, there is no detailed information provided on the causes of those mortalities. In the present study, mortality rate was found to be higher. The fact that grade 3 and 4 cases constituted the majority of the cases in our study may be the reason behind that. Linder et al. [25] conducted a study including 641 cases and lost $86(13.4 \%)$ of the premature newborns with LBW, whereas 36 of the cases in their study had grades 3 and 4 IVH and 27 (75\%) of those exhibited mortality. Kadri et al. performed a study in which they lost $86 \%$ of the cases with grade $3 \mathrm{GMH}$ and $100 \%$ of the cases with grade 4 GMH [7].

VSGS revision has been performed in $52(28 \%)$ of 185 cases by Tubbs et al. [17] and in $5(25 \%)$ of 20 cases by Fulmer et al. [5]. In both series, the only reason behind revision was the adhesion development in the subgaleal pouch, however, there was no obstruction in the intraventricular catheter. Particularly those adhesion scars are reported to arise from infected debris [5, 17, 21]. In revision surgery, in cases where the catheter is not obstructed, a simple dissection on the subgaleal pouch is reported to be enough [17]. In our series, revision was applied in three cases against subgaleal adhesions. These results show consistency with those in the literature.

In terms of needing no permanent VPS, while Willis et al. [9] reported a rate of $16.6 \%$ (one case), Fulmer et al. [5], Sklar et al. [18], and Rahman et al. [19] noted a rate of $20 \%$ (four cases), $11 \%$ (seven cases), and 20\% (three cases), respectively. In the current study, the rate of cases requiring VPS was $12 \%$ (three cases). Our result was consistent with the literature. 

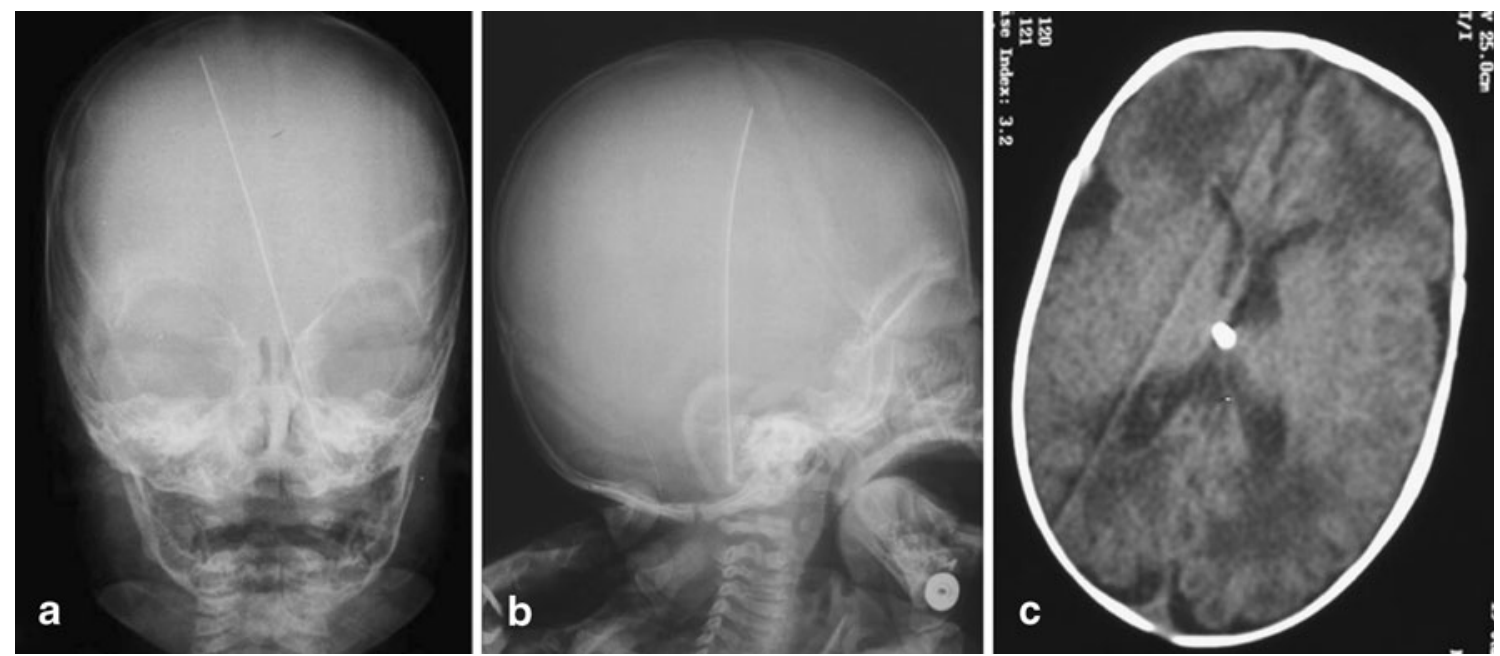

Fig. 9 Ventricular catheter into the fall and arrest, a case of hydrocephalus

Permanent VPS is reported to be necessary in the treatment of PHH generally seen in premature infants at a rate varying between 60 and $85 \%$ [24, 26-30]. While Willis et al. [9] found this rate as $83.4 \%$, Tubbs et al. [17], Fulmer et al. [5], Sklar et al. [18], and Rahman et al. [19] reported it as $84,75,90$, and $75 \%$, respectively. Moreover, shunt dependency occuring after other PHH treatment methods is reported to be $64-78 \%$ following EVD and $75-88 \%$ after subcutaneous reservoir use $[21,22,31,32]$. In the current study, shunt requirement was found to be $60 \%$. The low rate of shunt need is due to the loss of some cases with VSGS who needed VPS during the monitorization period.

The monitoring of PHH cases after VPS is reported to demonstrate $33 \%$ shunt infection complication by Fulmer et al. [5]. In our study, shunt infection rate was $24 \%$. Linder et al. [25] reported a mortality of $75 \%$ among cases with grades 3 and $4 \mathrm{GMH}$. Kadri et al. [7] noted the distribution of mortality rates over GMH grades as follows: $38 \%$ for grade $1,66 \%$ for grade $2,86 \%$ for grade 3 , and $100 \%$ for grade 4 . In the current study, while mortality rate among cases of grade 2 was $0 \%$, it was $38.5 \%$ for grade 3 and $50 \%$ for grade 4 cases. Those results were not consistent with the literature. The reason behind that inconsistency is the inadequacy of daily temporary CSF drainage applied through LPs until the VPS procedure against hydrocephalus in the study of Kadir et al. along with the contribution of VSGS in prognoses of our cases. In the following controls, we lost all our grade 4 cases in compliance with the

Table 1 Summary of results of other literature and our study

\begin{tabular}{|c|c|c|c|c|c|c|}
\hline & $\begin{array}{l}\text { Sklar et al. } \\
{[18]}\end{array}$ & $\begin{array}{l}\text { Rahman et al. } \\
\text { [19] }\end{array}$ & $\begin{array}{l}\text { Fulmer et al. } \\
{[5]}\end{array}$ & Tubbs et al. [17] & Willis et al. [9] & $\begin{array}{l}\text { Our work } \\
2007\end{array}$ \\
\hline Case number & 62 & 15 & 20 & 185 (71 PHH) & 6 & 25 \\
\hline Birth age (week) & 29.8 & & 29.1 & & 27.5 & 29.32 \\
\hline Birth weight (g) & 1,560 & & 1,724 & & 980 & 1,342 \\
\hline Preop head circumference $(\mathrm{cm})$ & 34.1 & & 33.6 & & 35.2 & 37.42 \\
\hline VSGS timing & & & 28 day (4 weeks) & & 30 day (4.2 weeks) & 33.08 day \\
\hline VSGS infection (\%) & 10 & & 0 & 5.9 & 66.7 & 8 \\
\hline CSF leakage (\%) & 32 & & 5 & 4.7 & 16 & 28 \\
\hline Intraparenchymal hemorrhage (\%) & & & 5 & 1.1 & & 0 \\
\hline VSGS died (\%) & 1.6 & & 20 & 9 & 16 & 28 \\
\hline Permanent shunt requirement (\%) & 90 & 80 & 75 & 84 & 83.4 & 60 \\
\hline VSGS stay (day) & & & 35.1 & 37.4 & & 44 \\
\hline VSGS revision & & & $5(25 \%)$ & 52 cases $(28 \%)$ & & $3(12 \%)$ \\
\hline VPS infection at follow & & & $5(33 \%)$ & & & $6(24 \%)$ \\
\hline VPS does not need permanent & $6(9 \%)$ & $3(20 \%)$ & $4(20 \%)$ & & $16.6 \%$ & $3(12 \%)$ \\
\hline
\end{tabular}


literature, whereas prognosis among our cases with grade 4 $\mathrm{GMH}$, was found to be poor.

Generally, survival rate of cases with GMH and IVH is reported to be $25-75 \%$ in the literature [33-35]. Kazan et al. [3] reported the mortality rate of IVH associated with GMH in premature newborns, with or without surgery, as $38 \%$. Kadri et al. [7] monitored 126 cases and reported 70 $(55.5 \%)$ losses. In the current study, when post-VPS period was also included in the monitorization, $52 \%$ (13 cases) were found to be lost during monitoring, which was a result consistent with the literature. All findings were compared with the literature in Table 1.

\section{Conclusion}

1. Rates of infections and their complications are lower than those of other PHH treatment methods in the literature.

2. If hydrocephalus persists when the BW exceeds $200 \mathrm{~g}$ in those cases, then VPS insertion is appropriate.

3. Performing VSGS without permanent VPS in cases with grades 1 and $2 \mathrm{GMH}$, can suffice in hydrocephalus treatment.

We believe, in order to provide a better quality of life and protect the weak cerebral tissues from the detrimental effects of hydrocephalus in newborns with grades 1,2, and 3 PHH, performing VSGS procedure would be appropriate.

Open Access This article is distributed under the terms of the Creative Commons Attribution Noncommercial License which permits any noncommercial use, distribution, and reproduction in any medium, provided the original author(s) and source are credited.

\section{References}

1. Papile LA, Burstein J, Burstein R, Koffler H (1978) Incidence and evolution of subependymal and intraventricular hemorrhage: a study of infants with birth weights less than $1,500 \mathrm{gm}$. J Pediatr 92(4):529-534

2. Reinprecht A, Dietrich W, Berger A, Bavinzski G, Weninger M, Czech T (2001) Posthemorrhagic hydrocephalus in preterm infants: long-term follow-up and shunt-related complications. Child's Nerv Syst 17:663-669

3. Kazan S, Güra A, Uçar T, Korkmaz E, Ongun H, Akyuz M (2005) Hydrocephalus after intraventricular hemorrhage in preterm and low-birth weight infants: analysis of associated risk factors for ventriculoperitoneal shunting. Surg Neurol 64:S2:77-S2:81

4. Horinek D, Cihar M, Tichy M (2003) Current methods in the treatment of posthemorrhagic hydrocephalus in infants. Bratisl Lek Listy 104(11):347-351

5. Fulmer BBMD, Grabb PAMD (2000) Neonatal ventriculosubgaleal shunts. Neurosurgery 47(1):80-84
6. Hudgins RJ, Boydston WR, Gilreath CL (1998) Treatment of posthemorrhagic hydrocephalus in the preterm infant with a ventricular access device. Pediatr Neurosurg 29:309-313

7. Kadri H, Mawla AA, Kazah J (2006) The incidence, timing, and predisposing factors of germinal matrix and intraventricular hemorrhage $(\mathrm{GMH} / \mathrm{IVH})$ in preterm neonates. Childs Nerv Syst 22:1086-1090

8. Cinalli G (1999) Alternatives to shunting. Childs Nerv Syst 15:718-731

9. Willis BK, Kumar CR, Wylen EL, Nanda A (2005) Ventriculosubgaleal shunts for posthemorrhagic hydrocephalus in premature infants. Pediatr Neurosurg 41:178-185

10. Mc Cullough D (1985) Hydrocephalus: treatment. In: Rengachary S, Wilkins R (eds) Neurosurgery, pp 2140-2150

11. Taylor AG, Peter JC (2001) Advantages of delayed VP shunting in post-haemorrhagic hydrocephalus seen in low-birth-weight infants. Child's Nerv Syst 17:328-333

12. Roland EH, Hill A (1997) Intraventricular hemorrhage and posthemorrhagic hydrocephalus. Current and potential future interventions. Clin Perinatol 24:589-605

13. Levy ML, Masri MS, McComb JG (1997) Outcome for preterm infants with germinal matrix hemorrhage and progressive hydrocephalus. Neurosurgery 41:1111-1118

14. Lin JP, Goh W, Brown JK, Steers AJW (1992) Neurological outcome following neonatal post-haemorrhagic hydrocephalus: the effects of maximum raised intracranial pressure and ventriculo-peritoneal shunting. Child's Nerv Syst 8:190-197

15. McCallum J, Turbeville D (1994) Cost and outcome in a series of shunted premature infants with intraventricular hemorrhage. Pediatr Neurol (1992) 20:63-67

16. Scarff TB, Anderson DE, Anderson CL (1992) Caldwell CC (1983) Complications of ventriculo-peritoneal shunts in premature infants. Concepts Pediatr Neurosurg 4:81-89

17. Tubbs RS, Smyth MD, Wellons JC, Blount JP, Grabb PA, Oakes WJ (2003) Life expectancy of ventriculosubgaleal shunt revisions. Pediatr Neurosurg 38:244-246

18. Sklar F, Adegbite A, Shapiro K, Miller K (1992) Ventriculosubgaleal shunts: management of posthemorrhagic hydrocephalus in premature infants. Pediatr Neurosurg 18:263-265

19. Rahman S, Teo C, Morris W, Lao D, Boop FA (1995) Ventriculosubgaleal shunt: a treatment option for progressive posthemorrhagic hydrocephalus. Childs Nerv Syst 11:650-654

20. Vinchon M, Lapeyre F, Duquennoy C, Dhellemmes P (2001) Early treatment of posthemorrhagic hydrocephalus in low-birthweight infants with valveless ventriculoperitoneal shunts. Pediatr Neurosurg 35:299-304

21. Bruinsma N, Stobberingh EE, Herpers MJ, Vles JS, Weber BJ, Gavilanes DA (2000) Subcutaneous ventricular catheter reservoir and ventriculoperitoneal drain-related infections in preterm infants and young children. Clin Microbiol Infect 6:202-206

22. Kim DK, Uttley D, Bell BA, Marsh HT, Moore AJ (1995) Comparison of rates of infection of two methods of emergency ventricular drainage. J Neurol Neurosurg Psychiatry 58:444-446

23. Richard E, Cinalli G, Assis D, Pierre-Kahn A, Lacaze-Masmonteil $\mathrm{T}$ (2001) Treatment of post-haemorrhage ventricular dilatation with an Ommaya's reservoir: management and outcome of 64 preterm infants. Child's Nerv Syst 17:334-340

24. Steinbok P, Cochrane DD (1994) Ventriculosubgaleal shunt in the management of recurrent ventriculoperitoneal shunt infection. Childs Nerv Syst 10:536-539

25. Linder N, Haksin O, Levit O, Klinger G, Prince T (2003) Risk factors for intraventricular hemorrhage in very low birth weight premature infants: a retrospective case-control study. Pediatrics 111:590-595

26. Gurtner P, Bass T, Gudeman S, Penix J, Philput C, Schinco F (1992) Surgical management of posthemorrhagic hydrocephalus in 22 low-birth-weight infants. Childs Nerv Syst 8:198-202 
27. Murphy BP, Inder TE, Rooks V, Taylor GA, Anderson NJ, Mogridge $\mathrm{N}$ et al (2002) Posthemorrhagic ventricular dilatation in the premature infant: natural history and predictors of outcome. Arch Dis Child Fetal Neonatal Ed 87:37-41

28. Tortorolo G, Luciano R, Papacci P, Tonelli T (1999) Intraventricular hemorrhage: past, present and future, focusing on classification, pathogenesis and prevention. Child's Nerv Syst 15:652-661

29. Ventriculomegaly Trial Group (1990) Randomised trial of early tapping in neonatal posthemorrhagic ventricular dilatation. Arch Dis Child 65:3-10

30. Cornips E, Van Calenbergh F, Plets C, Devlieger H, Casaer P (1997) Use of external drainage for posthemorrhagic hydrocephalus in very low birth weight premature infants. Child's Nerv Syst 13:369-374

31. Rhodes TT, Edwards WH, Saunders RL, Harbaugh RE, Little CL, Sargent SK (1987) External ventricular drainage for initial treatment of neonatal posthemorrhagic hydrocephalus: surgical and neurodevelopmental outcome. Pediatr Neurosci 13:255262

32. Robertson CM, Svenson LW, Joffres MR (1998) Prevalence of cerebral palsy in Alberta. Can J Neurol Sci 25:117-22

33. De Vries LS, Rademaker KJ, Groenendaal F, Eken P, van Haastert IC, Vandertop WP, Gooskens R, Meiners LC (1998) Correlation between neonatal cranial ultrasound, MRI in infancy and neurodevelopmental outcome in infants with a large intraventricular haemorrhage with or without unilateral parenchymal involvement. Neuropediatrics 29 (4): $180-188$

34. Hamrick SE, Miller SP, Leonard C, Glidden DV, Goldstein R, Ramaswamy V, Piecuch R, Ferriero DM (2004) Trends in severe brain injury and neurodevelopmental outcome in premature newborn infants: the role of cystic periventricular leukomalacia. J Pediatr 145(5):593-599

35. Pomerance JJ, Richardson J (1993) Neonatology for the clinician, 1st edn. Applenton \& Lange, Norwalk, pp 425-435 